\title{
A Million Element Integral Field Unit (MEIFU)
}

\author{
Simon Morris ${ }^{1}$, Robert Content ${ }^{1}$, Ray Sharples ${ }^{1}$, Richard Bower ${ }^{1}$, Roger \\ Davies $^{1}$, and Carlton Baugh ${ }^{1}$ \\ Physics Department, Durham University, South Rd., Durham, DH1 3LE, ENGLAND
}

\begin{abstract}
We describe an instrument concept that will provide simultaneous spectra for a million spatial samples on the sky. With the proposed field of view and spectral resolution, it will be able to measure redshifts and line strengths for around 2-4000 $\mathrm{z} \sim 3-7$ galaxies in a 16 night campaign. The main science driver is to obtain a complete census of the star formation properties of galaxies with $2.5<\mathrm{z}<6.7$ as a function of luminosity, environment, morphology and redshift. This survey will also allow us to study the evolution of the 3 dimensional power spectrum and test the connection between these early galaxies and the large scale structure of the Lyman $\alpha$ forest.

Additional science drivers include [OII] emitters with $0.13<\mathrm{z}<1.52$, abundance gradients in nearby galaxies, searches for planetary nebulae in nearby galaxy groups and clusters, lensed emission line objects, and spectroscopic searches for objects with continuum breaks. We expect that this instrument will make an ideal partner to the HST ACS, where ACS morphology and broadband colour information can complement the MEIFU spectroscopy and help remove any degeneracies in line identification.
\end{abstract}

\section{Introduction}

In this contribution to the workshop on 'Scientific Drivers for ESO Future VLT/VLTI Instrumentation', we would like to make a case for the study, construction and deployment of a wide-field, optical integral field unit (IFU). 'Wide field' here means around 5 arcminutes diameter, with 0.3 arcsecond sampling, and 'optical' means 420-940nm. As one might imagine, this implies a very large number of pixels in the final detector arrays (around $10^{9}$ ), but we feel that modern CCD mosaics are well enough understood to allow this. It also implies a large number of spectrographs. We estimate that the instrument will need 27 spectrographs. However, these spectrographs can be kept relatively simple, and 'mass production', or a modular approach, can be used to reduce the cost and risk of the individual units. This also allows the instrument to be deployed in a phased manner.

In this contribution, we start by discussing the context for such an instrument. We then describe the instrument concept and make some preliminary Signal-to-Noise (S/N) estimates. We can then combine these with semi-analytic calculations for the expected observed Lyman $\alpha$ emission from galaxies as a function of redshift (including the effects of dust obscuration), to give predictions for the numbers of detections. We conclude with some of the other possible science cases for the instrument. 
Table 1 lists some of the other facilities that will be demanding followup or complementary optical spectroscopy. As can be seen, many of these facilities have large fields of view (FOV).

Table 1. The need for complimentarity with other facilities

\begin{tabular}{ll}
\hline Facility & Field of View \\
\hline Chandra & $17 \times 17$ arcmin \\
XMM & 30 arcmin diameter \\
HST ACS & $3.3 \times 3.3$ arcmin \\
SIRTF & $5.1 \times 5.1$ arcmin \\
NGST Imaging & $4 \times 4$ arcmin \\
SCUBA & 2.3 arcmin diameter \\
ALMA & 12 arcsec diameter, but will mosaic fast \\
\hline
\end{tabular}

As demonstrated in Guy Monnet's introduction to the workshop, the VLT already has recognised this need, both with Multi-Object Spectrographs (MOS) with wide FOV, but very small filling factors within that FOV, and also with IFUs. We list some of these IFUs in table 2 in order to put the MEIFU proposal into context.

Table 2. (Some) optical 8m IFUs (wide field option)

\begin{tabular}{llll}
\hline Name & Telescope & FOV $(\operatorname{arcsec})$ & Sampling \\
\hline Flames & VLT & $11.5 \times 7.3$ & $17 \times 17$ \\
GMOS & Gemini & $5 \times 7$ & $32 \times 32$ \\
VIMOS & VLT & $54 \times 54$ & $80 \times 80$ \\
FMOS & Subaru & $15 \times(5 \times 5)$ & $15 \times(15 \times 15)$ \\
& & & \\
MEIFU & VLT & $300 \times 300$ & $1000 \times 1000$ \\
\hline
\end{tabular}

This will clearly require a quantum leap in IFU capabilities. We believe that this is possble, by using a combination of the lenslet developments from instruments such as OASIS and SAURON, together with a creative use of anamorphic magnification to improve the packing fraction on the detector. Both of these techniques are now well tested and understood. 


\section{Instrument Concept}

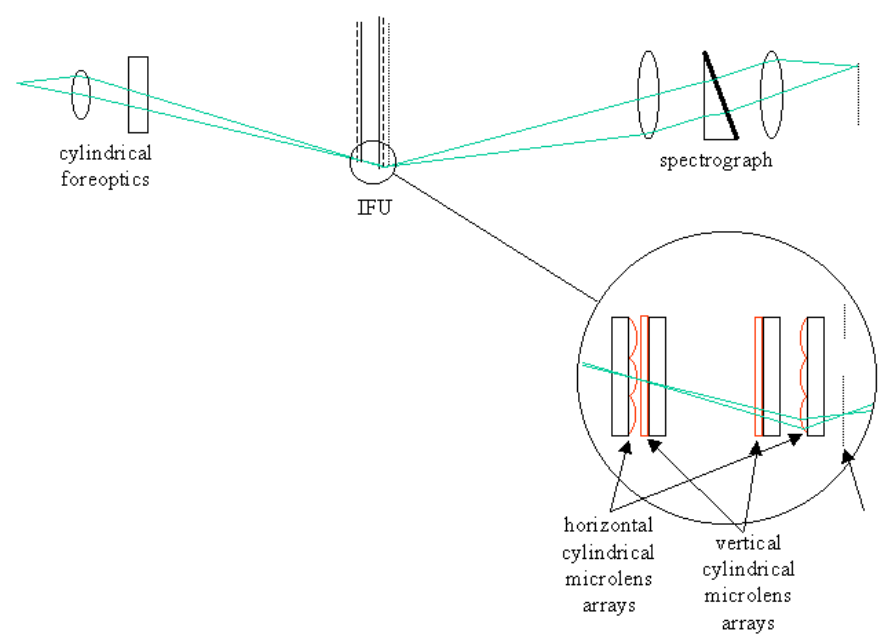

Fig. 1. Optical Principle: Fore-optics magnify the focal plane anamorphically (f/36 $x$ $\mathrm{f} / 195$ ) onto the first (rectangular) lenslet array which acts as the image slicer. The first cylindrical microlens arrays divide the input focal plane into micro-slices $(1.5 \mathrm{x} 0.3$ arcsec, 10x2 pixels). A second lenslet array anamorphically demagnifies the slices onto the input focal plane of the spectrograph and puts the pupil onto the grating.

The basic instrumental parameters are listed below, while the concept is explained in figures 1 and 2 .

- Approximately a $5 \times 5$ arcmin field of view

- $0.3 \times 0.15$ arcsec sampling (1000x2000 pixels)

- cover the entire wavelength range $420-940 \mathrm{~nm}$ in 4 exposures (by rotating the spectrograph FOV through 90 degrees each time)

- Spectral resolution (2 pixels) R 500 (420-750 nm), and 1500 (750-940 nm). The larger resolution in the red is needed because of the strong atmospheric OH emission.

- 27 spectrographs (12 covering 420-750nm, and 15 covering 750-940nm, each with $6 \mathrm{kx} 6 \mathrm{k}$ CCD mosaic detectors) 
- With a change in the fore-optics one could change the instrumental plate scale and convert the instrument to something optimised for Multi-Conjugate $\mathrm{AO}$.

- The intention is to use this instrument with an optical derotator and to mount it on the floor of a Naysmith platform.

- Detailed mass and costs budgets have not been developed, but BOTE estimates suggest that we can fit into the VLT mass limit and also within our guesses as to the available funding for second generation VLT facility instruments.

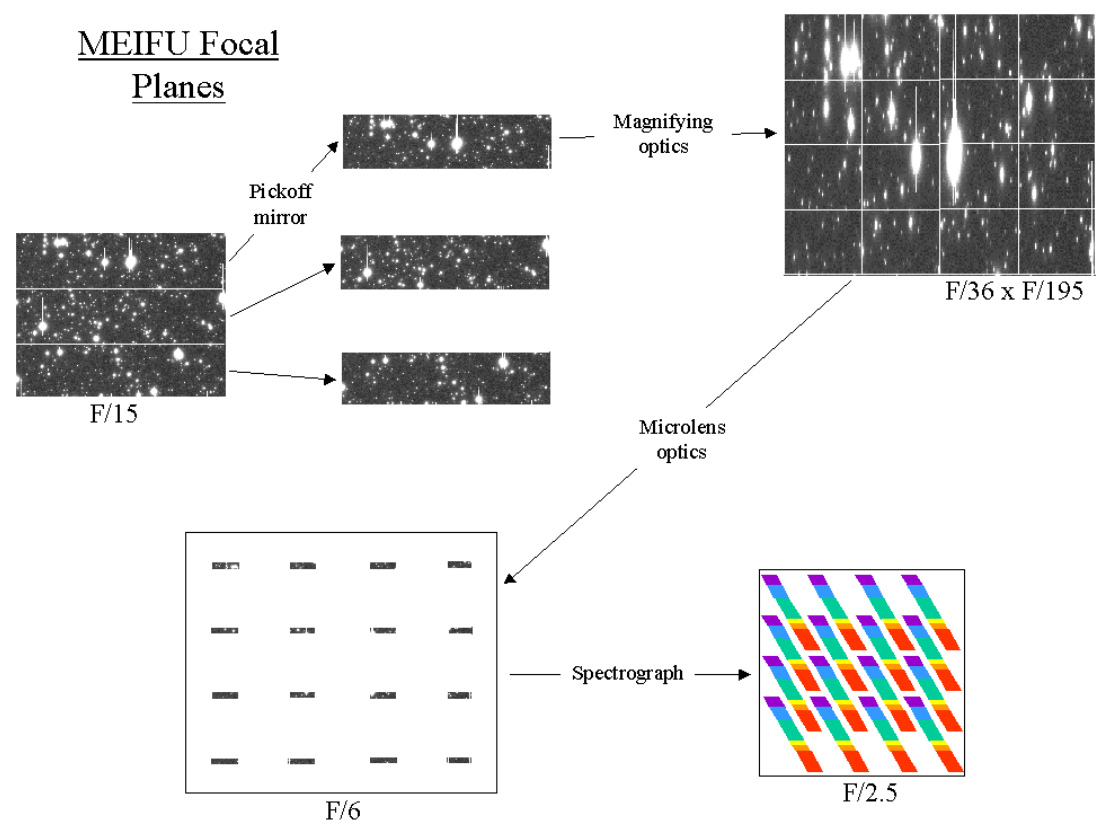

Fig. 2. MEIFU focal planes: Spectra are dispersed at an angle (13 deg) to avoid spectral overlap. The spectra are 200x12 pixels in the blue, and $675 \times 12$ pixels in the red on the detector ( 0.15 arcsec per pixel). Inter-spectrum gaps are 26 pixels (spectral) and 3 pixels (spatial)

We propose to use crossed cylindrical lenslet arrays made of fused silica and anti-reflection coated. The big advantage of this approach is that it is easy to make a lenslet array with different f-ratios in the $\mathrm{x}$ and $\mathrm{y}$ directions, i.e. it is naturally anamorphic.

Given the need for 27 spectrographs, reducing their cost and complexity is clearly crucial. Some of the key ideas we had to develop in order to do this were:

- de-magnifying the global pupil with the second lenslet arrays

- having non-parallel rays at Volume Phase Holographic (VPH) grating 
- only requiring 2 wavelength achromatism (helped by also only requiring a fairly small simultaneous wavelength coverage in any given spectrograph).

- maximising the use of off-the-shelf lenses

- splitting the field into 4 wavelength segments 420-510, 510-620, 620-750, 750$940 \mathrm{~nm}$, so spectrographs in each quadrant only cover one of these wavelength ranges.

This resulted in the spectrograph concept shown in figure 3 .

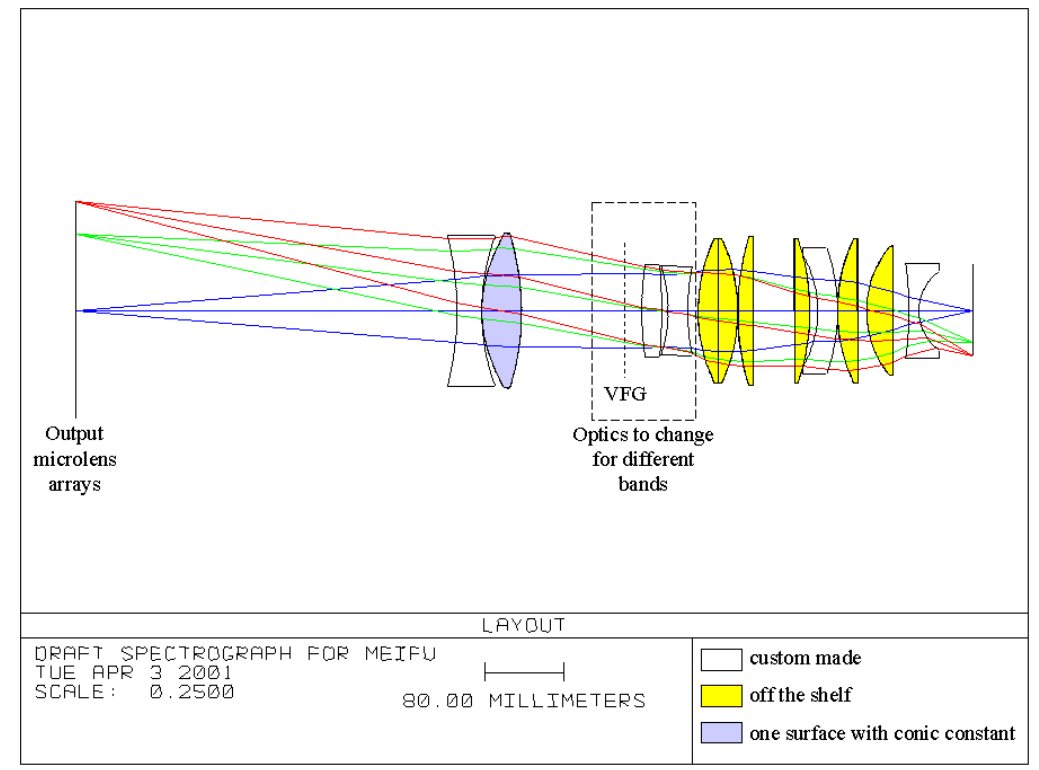

Fig. 3. Spectrograph concept: The design must be simple and low cost, as $27(12+15$ identical) are required. The effective field diameter is 16 arcmin. The camera focal ratio is $\mathrm{f} / 2.6$. The detector arrays are $6 \mathrm{k} \times 6 \mathrm{k} \times 15$ micron pixels, with $50 \% \mathrm{EE}$ in 1 pixel; $80 \%$ EE in 2 pixels. Note the extensive use of off the shelf lenses, and also the small number of optical elements that need to be changed in order to cover a different wavelength.

\section{Signal-to-Noise Estimates and Predicted Numbers of Detections}

With this instrument concept, we can now make plausible estimates for the $\mathrm{S} / \mathrm{N}$ achievable. We have tried to be conservative in these estimates, and to include all significant sources of noise. We list our assumptions and results below: 
- Assume an exposure time of $4 \times 8$ hours ( $10^{5}$ seconds), sky background in $\mathrm{V}=21.8$

- $27 \%$ sky-to-hard-disk throughput

- $50 \%$ of object flux in $0.6 x 0.6$ arcsec box. (This could probably be improved using optimal extraction techniques, but some of that gain may be lost due to the objects being resolved.)

- All of the line flux in 2 spectral pixels $(1.1 \mathrm{~nm})$

- This leads to a $5 \sigma$ detection limit of $9 \times 10^{-19} \mathrm{ergs} \mathrm{cm}^{-2} \mathrm{~s}^{-1}$ (which is a factor 22 fainter than current narrow band surveys, see table 3 ).

- At a z of 3 (with a resulting observed $\lambda$ of $486.3 \mathrm{~nm}$ ), this flux limit corresponds to a line luminosity of $7 \times 10^{40}$ ergs per second.

- We would need $4 \times 4$ nights to survey the entire 420-940 nm wavelength range $(2.45<\mathrm{z}<6.73)$, for the full $5 \times 5$ arcminute field, at this depth.

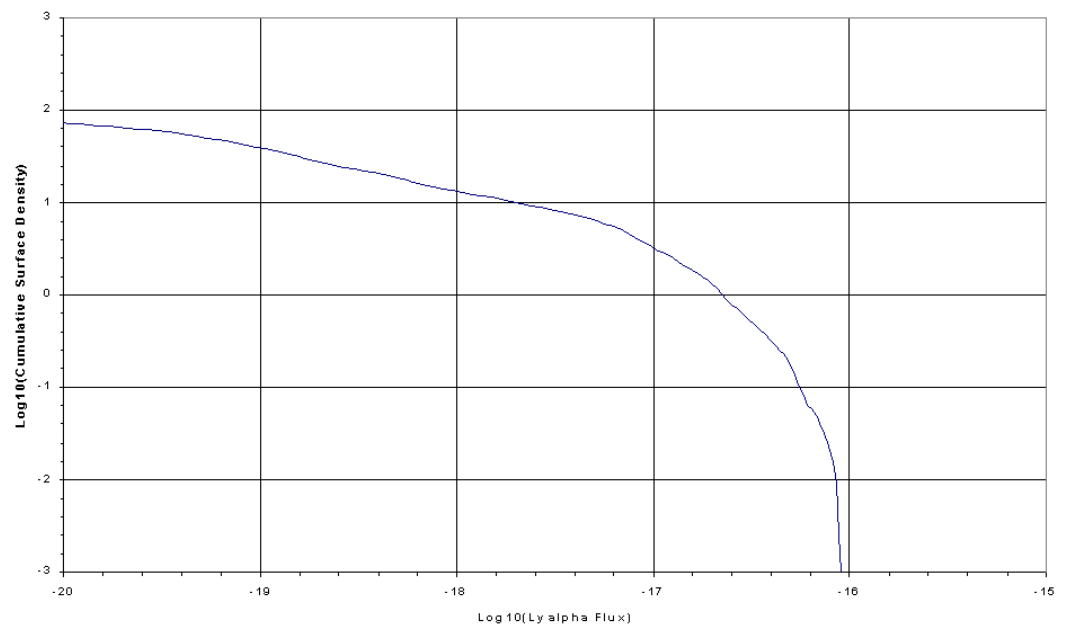

Fig. 4. Semi-analytic surface density prediction (having tuned the escape fraction to normalize to the observed counts at $3 \times 10^{-17}$ ergs per square $\mathrm{cm}$ per second). X-axis: $\log 10$ (Lyman $\alpha$ flux in ergs per square cm per second). Y-axis: Log10 (Number per square arcmin per deltaz $=1)$.

Now we would like to estimate roughly how many objects we might expect to detect in such a campaign. Since we are going substantially deeper than current data, we will have to rely on theoretical models to extrapolate down. For this we have used a semi-analytic model for galaxy formation (see [1] and references therein). These models predict a star formation rate (SFR), for each galaxy 
in the simulation, as a function of time. At the moment we are at liberty to tune the Lyman $\alpha$ escape fraction at will. We have chosen to assume that this is constant with redshift (probably conservative in that it is possible that the escape fraction may have been higher in the past due to lower metallicity of the objects). We then adjust the escape fraction to match the observed counts at the current flux limit around $2 \times 10^{-17} \mathrm{ergs}_{\mathrm{cm}}^{-2} \mathrm{~s}^{-1}$, and see what is predicted as we lower that flux limit. The counts do to start to flatten off, as can be seen in figure 4 , but nevertheless lead to a prediction of a factor 12 more objects per square arcminute.

The same models predict that the surface density of objects above some flux limit will fall fairly rapidly with redshift. As is shown in table 3, the current data do not strongly constrain this, but hint that the observed counts do not fall with redshift. Based on this we consider two cases - a pessimistic one where the numbers of object do indeed fall with redshift as predicted by the models, and an optimistic one where the numbers stay constant with redshift. It seems likely that the real situation is bracketed by these assumptions.

Table 3. Lyman $\alpha$ emitter surface densities from the literature

\begin{tabular}{lllll}
\hline z & flux lim (1) & Surface Density (2) & reference & year \\
\hline 2.42 & $3.00 \mathrm{E}-17$ & 1.3 & Stiavelli et al. & 2001 \\
2.81 & $3.70 \mathrm{E}-17$ & 1.0 & Warren \& Moller & 1996 \\
3.04 & $1.10 \mathrm{E}-17$ & 2.7 & Moller \& Fynbo & 2001 \\
3.09 & $3.00 \mathrm{E}-17$ & 2.3 & Steidel et al. & 2000 \\
3.15 & $2.00 \mathrm{E}-17$ & 4.2 & Kudritzki et al. & 2000 \\
3.43 & $3.00 \mathrm{E}-17$ & 2.7 & Cowie \& Hu & 1998 \\
3.43 & $1.50 \mathrm{E}-17$ & 3.7 & Cowie \& Hu & 1998 \\
3.43 & - & 6.9 & Cowie \& Hu & 1988 \\
4.5 & $2.00 \mathrm{E}-17$ & 1.1 & Rhoads et al. & 2001 \\
5 & - & 2.3 & Dawson et al. & 2001 \\
\hline
\end{tabular}

(1) Fluxes in ergs $\mathrm{cm}^{-2} \mathrm{~s}^{-1}$ (2) Surface densities per square arcminute per $\Delta \mathrm{z}$ of 1. Measurements of known overdensities were reduced by a factor 6 following Steidel et al. (2000).

From this, we can derive the number of objects we might expect to get in 16 nights. This is given in table 1 . We also show in figure 5 what is predicted for the spatial distribution of the objects within a single (4 night) exposure. 
Table 4. Predicted Numbers of detections in a 16 night exposure

\begin{tabular}{lrr}
\hline Z & Pessimistic & Optimistic \\
\hline $2.46-3.20$ & 773 & 773 \\
$3.20-4.10$ & 497 & 993 \\
$4.10-5.17$ & 303 & 1214 \\
$5.17-6.73$ & 207 & 1656 \\
& & \\
Total & 1780 & 4636 \\
\hline
\end{tabular}

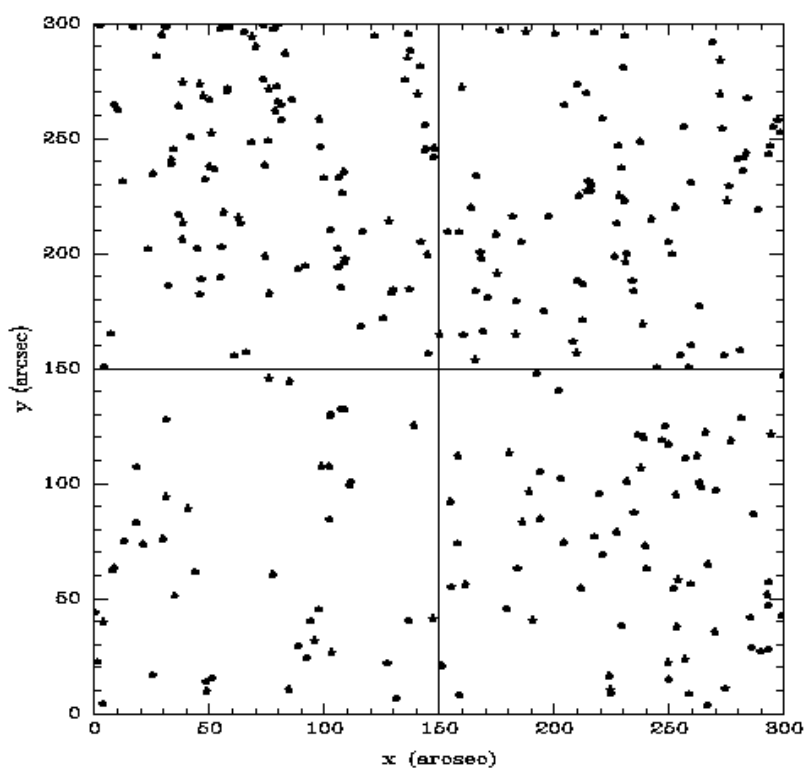

Fig. 5. Predicted surface density and angular distribution of 5- $\sigma$ detections in Lyman $\alpha$ from a 4 night exposure with MEIFU. Redshift ranges covered in each quadrant are: top-left $\mathrm{z}=2.46-3.20$, top-right $\mathrm{z}=3.20-4.10$, bottom-right $\mathrm{z}=4.10-5.17$, bottom-left $\mathrm{z}=5.17-6.73$. The proposed experiment would then rotate the spectrograph FOV by 90 , 180 and 270 degrees to obtain complete wavelength coverage for the whole FOV in 16 nights. 


\section{Conclusions}

With MEIFU we can both push to fainter luminosities and also cover a much larger continuous range in redshift than has been possible with narrow band surveys. Within the same data cube we will see:

- Lyman alpha emitters $(2.5<\mathrm{z}<6.75)$

- [OII] emitters $(0.13<\mathrm{z}<1.52)$

- faint $\mathrm{H}$ alpha emitters $(0<\mathrm{z}<0.43)$

Another science area for which this instrument will be ideal is mapping nearby galaxies, including:

- Starbursts (such as Messier 82)

- Measuring abundance gradients (e.g. comparing barred vs. unbarred galaxies)

- Looking at the stellar populations in local group dwarves (stellar spectral type and crude metallicities).

A few other candidate science cases that immediately suggest themselves are:

- A search for intergalactic planetary nebulae.

- Study of high velocity knots in galactic supernova remnants (searching for abundance anomalies).

- Study of nearby planetary nebulae (measuring variations in physical conditions across the nebula).

In analyses of this kind, the hardest benefit to quantify is serendipity. However, this is also, history teaches us, where the greatest strides forward occur. With its massive data collection capability and simultaneous coverage of volumes of space, we believe MEIFU will be a serendipity factory. Mining the deep MEIFU data cubes will probably turn up many exciting and interesting surprises.

We would like to acknowledge considerable help and advice from R. Bacon and the other members of the SAURON collaboration.

\section{References}

1. A. J. Benson, A. Nusser, N. Sugiyama, C. G. Lacey, 2001, MNRAS, 320, 153

2. L. L. Cowie, E. M. Hu, 1998, A.J., 115, 1319

3. S. Dawson, D. Stern, A. J. Bunker, H. Spinrad, A. Dey, 2001, astro-ph/0105043

4. R. P. Kudritzki et al., 2000, Ap. J., 536, 19

5. P. Moller, J. U. Fynbo, 2001, astro-ph/0105114

6. J. E. Rhoads, S. Malohatra, A. Dey, B. T. Jannuzi, D. Stern, H. Spinrad, 2001, astro-ph/0104294.

7. C. C. Steidel, K. L. Adelburger, A. E. Shapley, M. Pettini, M. Dickinson, M. Giavalisco, 2000, Ap. J., 532, 170

8. M. Stiavelli, et al., 2001, astro-ph/0105503

9. S. J. Warren, P. Moller, 1996, A.\&A., 311, 25 